\title{
Automated Construction using Contour Crafting - Applications on Earth and Beyond
}

\author{
by \\ B. Khoshnevis, Professor, Industrial \& Systems Engineering \\ George Bekey, Professor, Computer Science \\ University of Southern California \\ khoshnev@usc.edu, bekey@usc.edu
}

\begin{abstract}
Although automation has advanced in manufacturing, the growth of automation in construction has been slow. Conventional methods of manufacturing automation do not lend themselves to construction of large structures with internal features. This may explain the slow rate of growth in construction automation. Contour Crafting (CC) is a recent layered fabrication technology that has a great potential in automated construction of whole structures as well as sub-components. Using this process, a single house or a colony of houses, each with possibly a different design, may be automatically constructed in a single run, imbedded in each house all the conduits for electrical, plumbing and air-conditioning. Our research also addresses the application of $\mathrm{CC}$ in building habitats on other planets. $\mathrm{CC}$ will most probably be one of the very few feasible approaches for building structures on other planets, such as Moon and Mars, which are being targeted for human colonization before the end of the new century.
\end{abstract}

KEYWORDS: Contour Crafting, housing construction, construction on other planets

\section{INTRODUCTION}

Since the early years of the twentieth century automation has grown and prevailed in almost all production domains other than construction of civil structures. Implementation of automation in the construction domain has been slow due to: a) unsuitability of the available automated fabrication technologies for large scale products, b) conventional design approaches that are not suitable for automation, c) significantly smaller ratio of production quantity/type of final products as compared with other industries, d) limitations in the materials that could be employed by an automated system, e) economic unattractiveness of expensive automated equipment, and f) managerial issues. On the other hand, the following are reported to be serious problems that the construction industry is facing today (Warszawski and Navon, 1998):
- Labor efficiency is alarmingly low,

- Accident rate at construction sites is high,

- Work quality is low, and

- Control of the construction site is insufficient and difficult, and skilled workforce is vanishing. 
Automation of various parts and products has evolved considerably in the last two centuries but construction remains largely as a manual practice. This is because the various conventional methods of manufacturing automation do not lend themselves to construction of large structures. A promising new automation approach is layered fabrication, generally known as rapid prototyping. Although several methods of rapid prototyping have been developed in the last two decades (Pegna, 1997), and successful applications of these methods have been reported in a large variety of domains (including industrial tooling, medical, toy making, etc.), currently Contour Crafting (CC) seems to be the only layer fabrication technology that is uniquely applicable to construction of large structures such as houses (Khoshnevis, 2000).

\section{CONTOUR CRAFTING}

Contour Crafting (CC) is an additive fabrication technology that uses computer control to exploit the superior surface-forming capability of troweling to create smooth and accurate planar and free-form surfaces (Khoshnevis 1998, Khoshnevis et al., 2001-a; Khoshnevis et al, 2001-b). Some of the important advantages of $\mathrm{CC}$ compared with other layered fabrication processes are better surface quality, higher fabrication speed, and a wider choice of materials.
The key feature of CC is the use of two trowels, which in effect act as two solid planar surfaces, to create surfaces on the object being fabricated that are exceptionally smooth and accurate. Artists and craftsmen have effectively used simple tools such as trowels, blades, sculpturing knives, and putty knives, shown in Figure 1, with one or two planar surfaces for forming materials in paste form since ancient times. Their versatility and effectiveness for fabricating complex free-form as well as planar surfaces is evidenced by ancient ceramic containers and

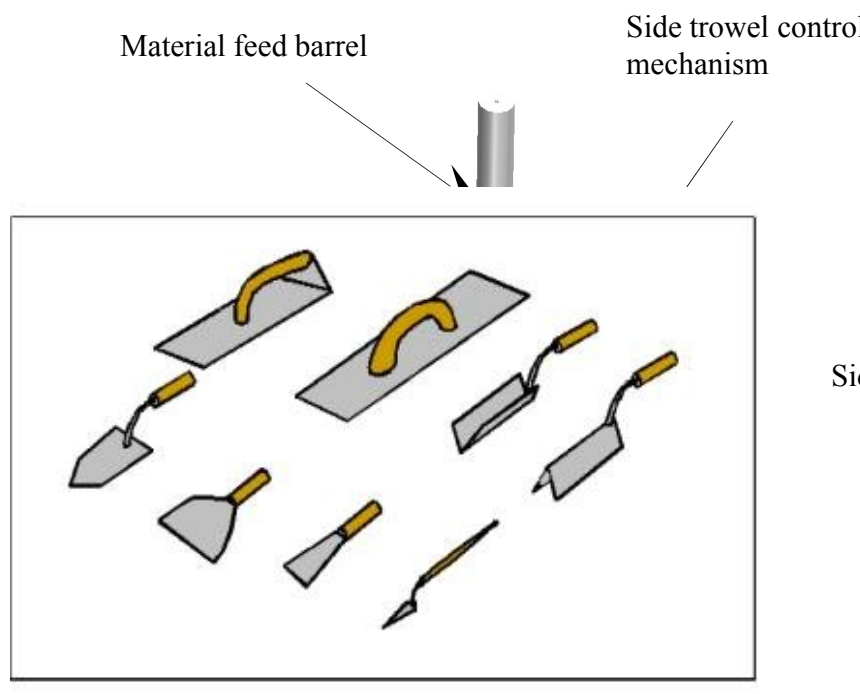

Figure 1. Simple Historical Construction Tools

sculptures with intricate or complex surface geometries as well as detailed plaster work that have shapes as complicated as flowers, on the walls of rooms. Surface shaping knives are used today for industrial model making (e.g., for building clay models of car bodies). However, despite the progress in process mechanization with computer numerical control and robotics, the method of using these simple but powerful tools is still manual, and their use is limited to model building and plaster work in construction. 
In CC, computer control is used to take advantage of the superior surface forming capability of troweling to create smooth and accurate, planar and free-form surfaces. The layering approach enables the creation of various surface shapes using fewer different troweling tools than in traditional plaster handwork and sculpting. It is a hybrid method that combines an extrusion process for forming the object surfaces and a filling process (pouring or injection) to build the object core. As shown in Figure 2, the extrusion nozzle has a top and a side trowel. As the material is extruded, the traversal of the trowels creates smooth outer and top surfaces on the layer. The side trowel can be deflected to create non-orthogonal surfaces. The extrusion process builds only the outside edges (rims) of each layer of the object. After complete extrusion of each closed section of a given layer, if needed filler material such as concrete can be poured to fill the area defined by the extruded rims.

\section{APPLICATION IN CONSTRUCTION}

Aplication of $\mathrm{CC}$ in building construction is depicted in Figures 3 where a gantry system carrying the nozzle moves on two parallel lanes installed at the construction site. A single house or a colony of houses, each with possibly a different design, may be automatically constructed in a single run. Conventional structures can be built by integrating the CC machine with a support beam picking and positioning arm, and adobe structures, such the ones designed by CalEarth (www.calearth.org) and depicted in the Figure 4, may be built without external support elements using shape features such as domes and vaults. Following are some interesting aspects of this automated construction concept:

Design Flexibility: The process allows architects to design structures with functional and exotic architectural geometries that are difficult to realize using the current manual construction practice.
Multiple Materials: Various materials for outside surfaces and as fillers between surfaces may be used in CC. Also, multiple materials that chemically react with one another may be fed through the CC nozzle system and mixed in the nozzle barrel immediately before deposition. The quantity of each material may be controlled by computer and correlated to various regions of the geometry of the structure being built. This

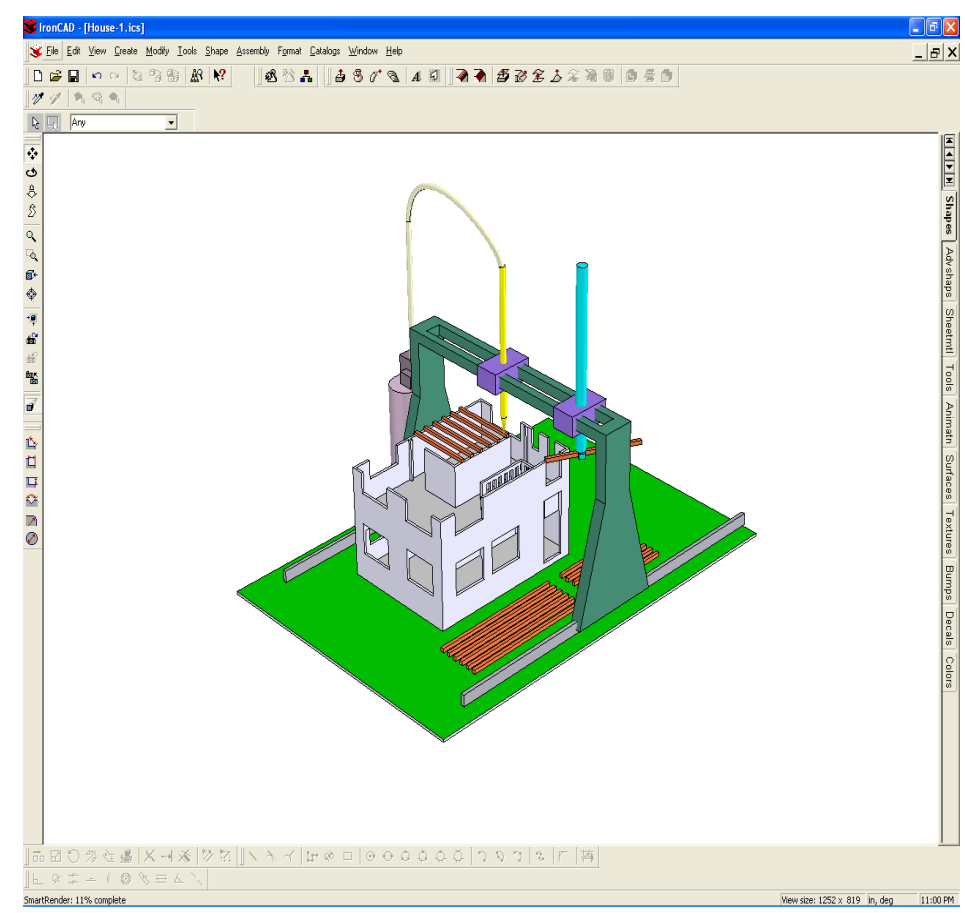

Figure 3.Construction of conventional buildings using CC

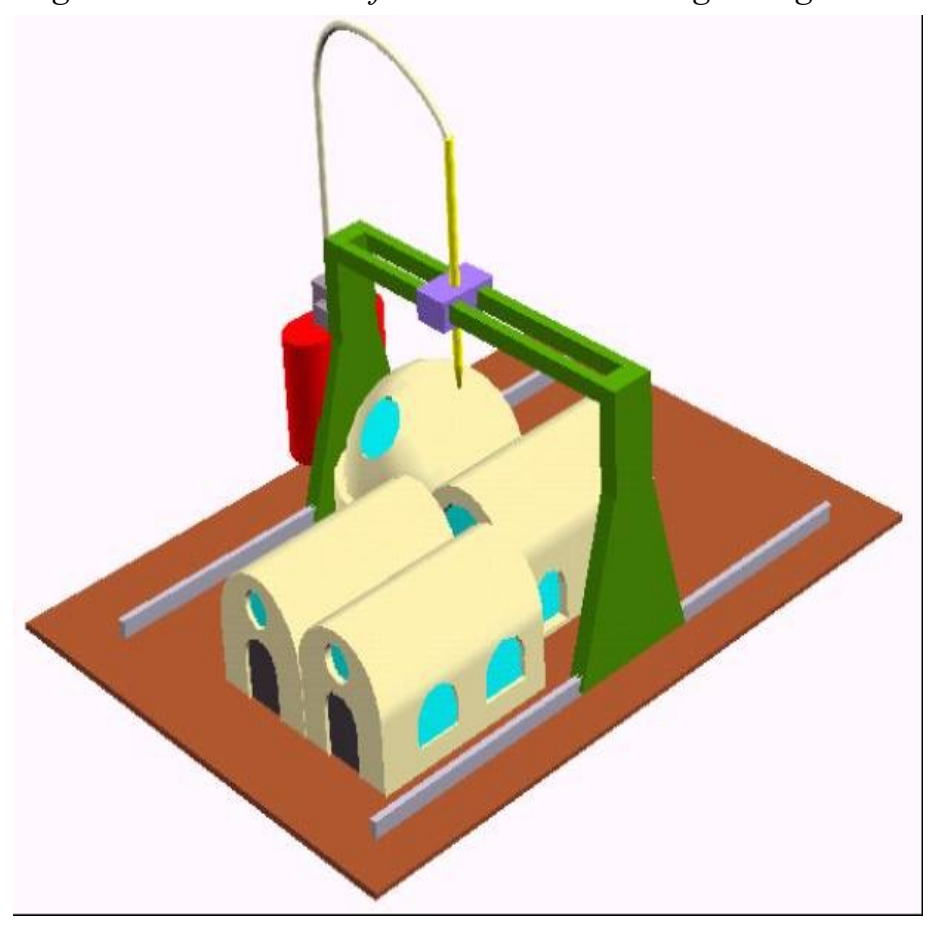


will make possible the construction of structures that contain varying amounts of different compounds in different regions.

Utility Conduits: As shown in Figure 5 utility conduits may be built into the walls of a building structure precisely as dictated by the CAD data. Sample sections made with CC and filled with concrete as shown in Figure 8 demonstrate this possibility.

Paint-Ready Surfaces: The quality of surface finish in CC is controlled by the trowel surface and is independent of the size of the nozzle orifice. Consequently, various additives such as sand, gravel, reinforcement fiber, and other applicable materials available locally may be mixed and extruded through the $\mathrm{CC}$ nozzle. Regardless of the choice of materials, the surface quality in $\mathrm{CC}$ is such that no further surface preparation would be needed for painting surfaces. Indeed an automated painting system may be integrated with CC.

Smart Materials: Since deposition in $\mathrm{CC}$ is controlled by computer, accurate amounts of selected construction materials, such as smart concrete, may be deposited precisely in the intended locations. This way the electric resistance, for example, of a carbon filled concrete may be accurately set as dictated by the design. Elements such as strain sensors, floor and wall heaters can be built into the structure in an integrated and fully automated manner.
Reinforcement: Modular imbedding of steel mesh reinforcement into each layer may be devised, as shown in Figure 6. The two simple modular components shown in this figure may be delivered by an automated feeding system that deposits and assembles them between the two rims of each layer built by CC. Concrete may then be poured between the rims of each layer to contain the steel mesh. The mesh can follow the geometry of the structure. Note that in this configuration the $\mathrm{CC}$ nozzle, the steel feeder, and the concrete filler feeder are all on the same gantry system. Such a system can create shapes with smooth outer surfaces and reinforced internal structure automatically and in one setup. 


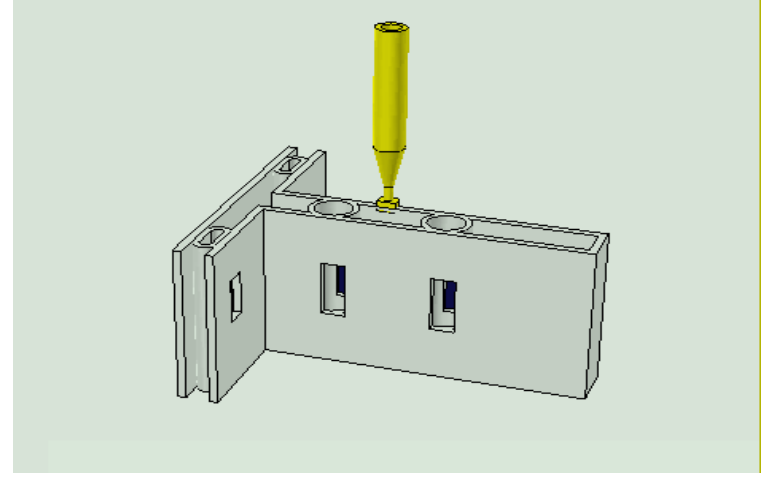

Figure 5. Complex Wall Section

As an alternative to traditional metal reinforcement, other advanced materials can be used, such as the fiber reinforced plastics (FRP). Since the nozzle orifice in $\mathrm{CC}$ does not need to be very small, it is possible to feed glass or carbon fiber tows through the $\mathrm{CC}$ nozzle to form continuous reinforcement consolidated with the matrix materials to be deposited. In the proposed study, deposition of the FRP reinforcement by a parallel nozzle built into the $\mathrm{CC}$ nozzle assembly will also be considered. Co-extrusion is further discussed in a later section.

Reinforcement can also be provided using the post-tensioning system. Accurate ducts can be generated by the $\mathrm{CC}$ process. Similar to posttensioned concrete construction, metal or FRP wires can be fed through the ducts and then post-tensioned to provide reinforcement.

\section{STATE OF DEVELOPMENT AND FUTURE PLAN}

Several CC machines have been developed at USC for research on fabrication with various materials including thermoplastics, thermosets, and various types of ceramics. These machine include a XYZ gantry system, a nozzle assembly with three motion control components (extrusion, rotation, and trowel deflection) and a six axis coordinated motion control system. The machine developed for ceramics processing is capable of extruding a wide variety of materials including clay and concrete.

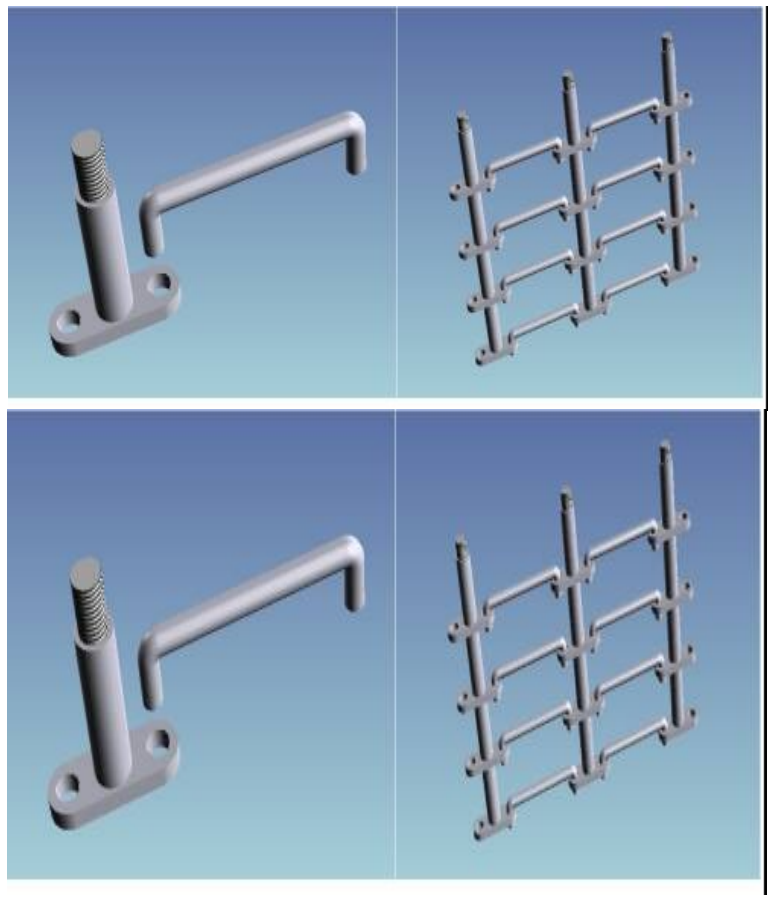

Figure 6. Steel reinforcement modules and progressively constructed reinforcement mesh

We have conducted extensive experiments to optimize the CC process to produce a variety of $2.5 \mathrm{D}$ and $3 \mathrm{D}$ parts with square, convex, and concave features, some filled with concrete, as shown in Figures 7 and 8 . The scale has been of the samples made to date (the hand in Figure 7 is indicative of the scale. We are currently working on the development of new nozzle assemblies that are especially designed for construction application. With the new nozzles we intend to first fabricate full scale sections of various building features such as sections of walls with conduits built in, and supportless roofs and perform various structural analysis and testing using a wide variety of candidate materials.

We plan to consider the NIST RoboCrane system as an alternative to a conventional $\mathrm{XYZ}$ gantry which may encounter problems due to rail alignment and structural rigidity. 
We plan to explore the applicability of the CC technology for building habitats on the Moon and Mars. In the recent years there has been growing interest in the idea of using these planets as platforms for solar power generation, science, industrialization, exploration of our Solar System and beyond, and for human colonization. In particular, the moon has been
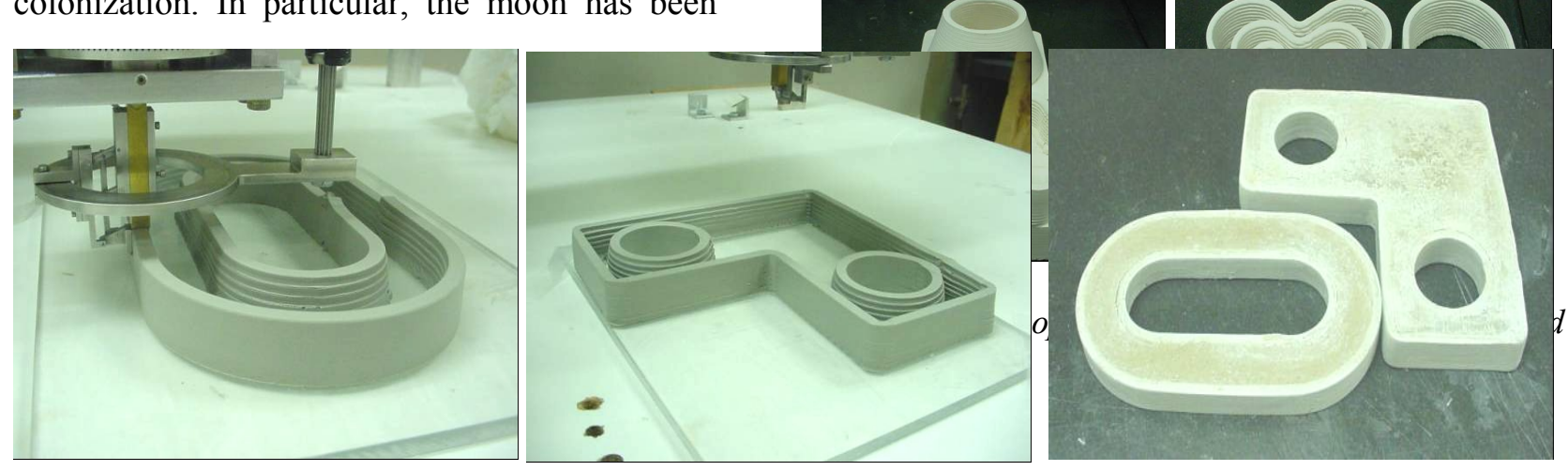

Figure 8. Sections with cavities made with CC and filled with concrete (largest dimension in these figures is 10")

suggested as the ideal location for solar power generation (and subsequent microwave transmission to earth via satellite relay stations). A conference on Space Solar Power sponsored by NASA and NSF (and organized by USC faculty) included several papers on this topic (http://robotics.usc.edu/workshops/ssp2000/).

Construction of solar collectors may be possible by using robots to assemble panels of photovoltaic cells shipped from earth. However, the cost of shipping these panels may be prohibitive. In fact, the materials needed to manufacture phovoltaic cells are all present in the lunar regolith, so that it may be more practical to build a "factory", perhaps by Contour Crafting technology, rather than shipping panels from earth. Furthermore, once solar power is available, it should be possible to adapt the current Contour Crafting technology to the lunar and other environments to use this power and in situ resources to build various forms of infrastructures such as roads and buildings. The lunar regolith, for example, may be used as the construction material. Other researchers have shown that lunar regolith can be sintered using microwave to produce construction materials such as bricks. We envision a Contour Crafting system that uses a limited amount of water to form a small batch of the lunar material into paste form. Once extruded and deposited in the desirable location, the water could be extracted and recycled for the next batch of material. Microwave sintering using solar power can be integrated into the system and hence progressively cured structures of various complexities could be built.

One of the ultimate goals of the Human Exploration and Development of Space (HEDS) program of NASA is colonization, i.e., building habitats for long term occupancy by humans. We believe that the Contour Crafting technology is the ideal method for such construction.

ACKNOWLEDGEMENT: This material is based upon work supported by the National Science Foundation under Grants No. 9522982 and 9615690, and by a grant from Office of Naval Research.

\section{REFERENCES}


Balaguer C., M. Abderrahim, S. Boudjabeur, P. Aromaa, K. Kahkonen, S. Slavenburg, D. Seward, T. Bock, R. Wing, \& B. Atkin (2002), "FutureHome: An Integrated Construction Automation Approach," IEEE Robotics \& Automation Magazine, pp 55-66.

Khoshnevis, B. (1998), "Innovative rapid prototyping process makes large sized, smooth surfaced complex shapes in a wide variety of materials", Materials Technology, Vol. 13, No. 2, pp. 52-63.

Khoshnevis, B., Russell, R., Kwon, H., \& Bukkapatnam, S. (2001-a), "Contour Crafting A Layered Fabrication Technique", Special Issue of IEEE Robotics and Automation Magazine, 8:3, pp 33-42.

Khoshnevis, B., Bukkapatnam, S., Kwon, H., \& Saito, J. (2001-b), "Experimental Investigation of Contour Crafting using Ceramics Materials", Rapid Prototyping J., Vol. 7, No.1, pp. 32-41.

Kulkarni, P., and Dutta, D. (1999), "Deposition Strategies and Resulting Part Stiffnesses in Fused Deposition Modeling", Journal of Manufacturing Science and Engineering, ASME, No.1, pp. 93-103, February.

Pegna, J. (1997), "Explorary investigation of solid freeform construction", Automation in construction, Vol. 5, No. 5, pp. 427-437.

Shrunk, D. Sharpe, B, Cooper, C., and Thangavelu, M., (1999), The Moon: Resources, Future Developments, and Colonization, WileyPraxis Series in Space Science and Tech., NY.

Warszawski, A., and Navon, R. (1998) "Implementation of robotics in buildings: current status and future prospects," Journal of Construction Engineering and Management, Vol.124, No.1, pp. 31-41.

Zak, G., Sela, M. N., Park, C. B., and Benhabib, B. (1999), "Layered-Manufacturing of FiberReinforced Composites", Journal of Manufacturing Science and Engineering, ASME, Vol. 121, pp. 448-455, August. 